\title{
Multiple Congenital Pancreatic Cysts in a Neonate
}

\author{
Eun Mi Kwon ${ }^{1}$, Jaeho Shin ${ }^{2}$, and Ga Won Jeon ${ }^{1}$ \\ ${ }^{1}$ Department of Pediatrics, ${ }^{2}$ Division of Pediatric Surgery, Department of Surgery, Inje University Busan Paik Hospital, Inje University \\ College of Medicine, Busan, Korea
}

\section{ABSTRACT}

Congenital pancreatic cysts are rare in newborn babies, and this makes prenatal diagnosis difficult. Diagnosis can be delayed for a few months after birth until the infant presents with an abdominal mass, abdominal distension, or vomiting due to gastric outlet obstruction. Excision of the cyst is the treatment of choice. A congenital pancreatic cyst should be considered if the fetus has an abdominal cyst without a definite origin. A prompt diagnosis is crucial to prevent fatal complications such as cholangitis, pancreatitis, cyst rupture, and peritonitis. We report a case of neonate with multiple congenital pancreatic cysts suspected prenatally to be stomach diverticulum or duplication cysts of the intestine.

Key Words: Congenital; Pancreatic cyst; Pancreatic pseudocyst; Infant, newborn

\section{INTODUCTION}

Congenital pancreatic cysts are a rare condition ${ }^{1)}$. Sometimes, pancreatic pseudocysts are caused by trauma to the pancreas or pancreatitis. A true congenital pancreatic cyst is extremely rare in newborn babies ${ }^{2}$. This type of cyst can be caused by developmental abnormalities of the pancreatic duct, and the resulting sequestration of the primitive pancreatic duct $^{1-4)}$. Prenatal diagnosis is difficult due to their extreme rarity. Therefore, the diagnosis of a congenital pancreatic cyst can be delayed until a few months after birth, when the infant presents with an abdominal mass, abdominal distension, or vomiting due to a gastric outlet obstruction. Excision of the cyst is the treatment of choice ${ }^{3)}$.

Multiple congenital pancreatic cysts may be accompanied by systemic diseases or other congenital conditions such as von Hippel-Lindau syndrome, polycystic kidney disease, Ivemark syndrome, trisomy 13, trisomy 15, and Meckel-Gruber syndrome ${ }^{5 \text { ) }}$. Cystic fibrosis can also lead to cystic dilatation of pancreatic ducts ${ }^{5}$. A congenital pancreatic cyst should be considered if the fetus has an abdominal cyst without a definite origin. Early diagnosis with evaluation of the accompanying disease is required. A prompt diagnosis is crucial to prevent fatal complications such as cholangitis, pancreatitis, cyst rupture, and peritonitis ${ }^{6}$. This case report discusses a newborn infant with multiple pancreatic cysts diagnosed by magnetic resonance cholangio-pancreatography (MRCP). These cysts were suspected prenatally to be stomach diverticulum or duplication cysts of the intestine by fetal ultrasound.
Received: 30 August 2018

Revised: 22 January 2019

Accepted: 29 January 2019

Correspondence to: Ga Won Jeon

Department of Pediatrics, Inje University Busan Paik Hospital, Inje University College of Medicine, 75 Bokji-ro, Busanjingu, Busan 47392, Korea

Tel: +82-51-890-6497

Fax: +82-51-895-7785

E-mail: iamgawon@hanmail.net https://orcid.org/0000-0002-8206-9727

Copyright(c)

By Korean Society of Neonatology.

All right reserved.

This is an Open-Access article distributed under the terms of the Creative Commons Attribution Non-Commercial License (http://creativecommons.org/licenses/ by-nc/4.0), which permits unrestricted non-commercial use, distribution, and reproduction in any medium, provided the original work is properly cited. 


\section{CASE REPORT}

This study was approved by the Institutional Review Board of Busan Paik Hospital (19-0030), and a waiver of consent was granted for chart review without patient contact.

A 35-year-old primigravida woman was referred to our medical center for further evaluation of fetal intraabdominal cysts in a twin baby. A prenatal ultrasonography in our medical center suspected these cysts to be stomach diverticulum or duplication cysts of the intestine (Figure 1).

At $36^{+5}$ weeks, a cesarean delivery was performed and male twins were born. The weight of the first baby was $2.9 \mathrm{~kg}$ (50th percentile). However, the weight of the second baby was $1.9 \mathrm{~kg}$ ( $<3$ rd percentile). The second baby was much smaller than the first, and thus, he was hospitalized in the neonatal intensive care unit. His height was $45 \mathrm{~cm}$ (3rd to 10th percentile) and his head circumference was $31.5 \mathrm{~cm}$ (10th to 25th percentile). His Apgar score was 6 at 1 minute and 8 at 5 minutes. Direct bilirubin and >-glutamyl-transferase (GGT) started to elevate 2 weeks after birth. However, he did not show signs of hemolytic anemia causing direct hyperbilirubinemia. His direct bilirubin level was $2.0 \mathrm{mg} / \mathrm{dL}$, with a total bilirubin level of $4.9 \mathrm{mg} / \mathrm{dL}$, GGT level of $240 \mathrm{U} / \mathrm{L}$ (reference, 13 to 147), and alkaline phosphatase (ALP) level of $634 \mathrm{U} / \mathrm{L}$ (reference, 145 to 420 ). His serum glucose level

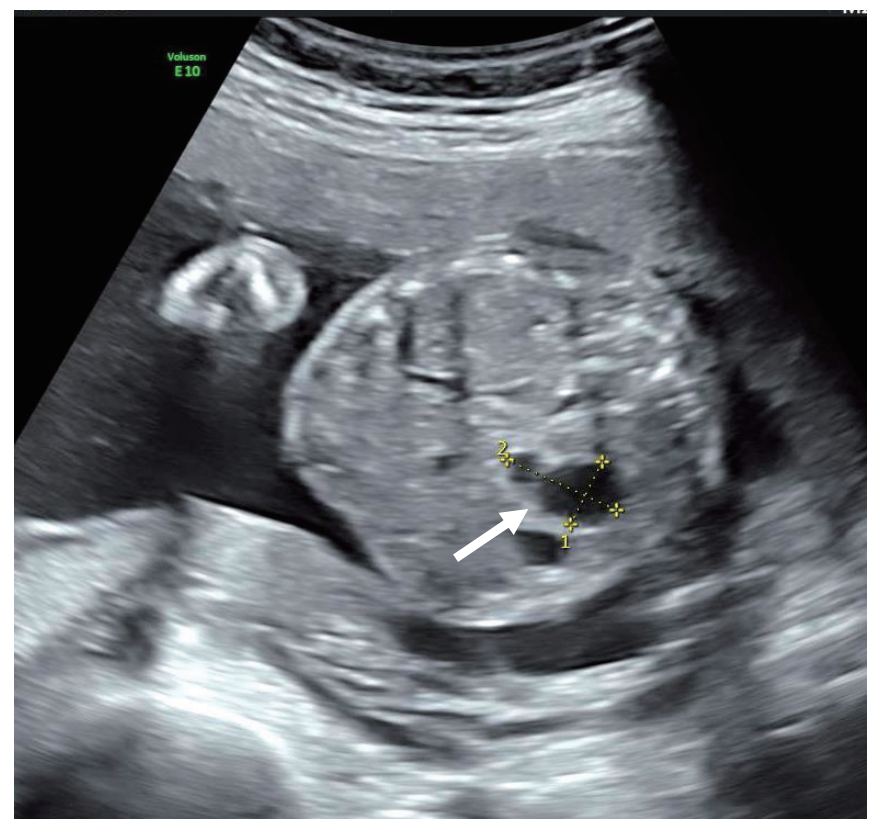

Figure 1. Prenatal ultrasonography of the second fetus with abdominal cysts. Prenatal ultrasonography showing stomach diverticulum or duplication cysts of the intestine $10.6 \times 18.2 \mathrm{~mm}$ in diameter (arrow). of 70 to $90 \mathrm{mg} / \mathrm{dL}$ was normal (reference, 50 to 90 ). We conducted an abdominal ultrasonography at the 16th postnatal day that showed a collapsed gallbladder, strongly suggestive of congenital biliary atresia (Figure 2). An MRCP was performed to confirm congenital biliary atresia on the 22nd postnatal day; it showed multiple, variable sized cysts in the pancreatic head and tail, with the largest one (16 $\mathrm{mm}$ in diameter) located in the pancreatic tail (Figure 3). The levels of amylase and lipase were normal: amylase, $6 \mathrm{IU} / \mathrm{L}$; lipase, $14 \mathrm{U} / \mathrm{L}$.

To investigate associated congenital malformations, further evaluations were performed. The echocardiogram findings were normal, other than the detection of a small atrial septal defect $2.6 \mathrm{~mm}$ in size. There were no abnormalities in the cranial, renal, or sacral ultrasonography. A chromosome analysis showed a normal male karyotype.

We started treatment using ursodoxycholic acid, and then direct bilirubin and GGT started to decrease and normalized at discharge from the hospital (direct bil irubin, $0.8 \mathrm{mg} / \mathrm{dL}$; GGT, 57 $\mathrm{U} / \mathrm{L}$ ). The total bilirubin level was $2.7 \mathrm{mg} / \mathrm{dL}$ and ALP was 1,029 $\mathrm{U} / \mathrm{L}$. As discussed above, his amylase and lipase levels had been normal, and were $11 \mathrm{IU} / \mathrm{L}$ and $10 \mathrm{U} / \mathrm{L}$ at discharge, respectively.

The baby was discharged on the 34th postnatal day. He was clinically well without jaundice or an acholic stool. Exploratory laparotomy or excision of cysts had not been performed yet. He is still under regular radiologic and laboratory checkups for pancreatic and hepatic enzymes. If either of these findings are aggravated, we will proceed with an operation.

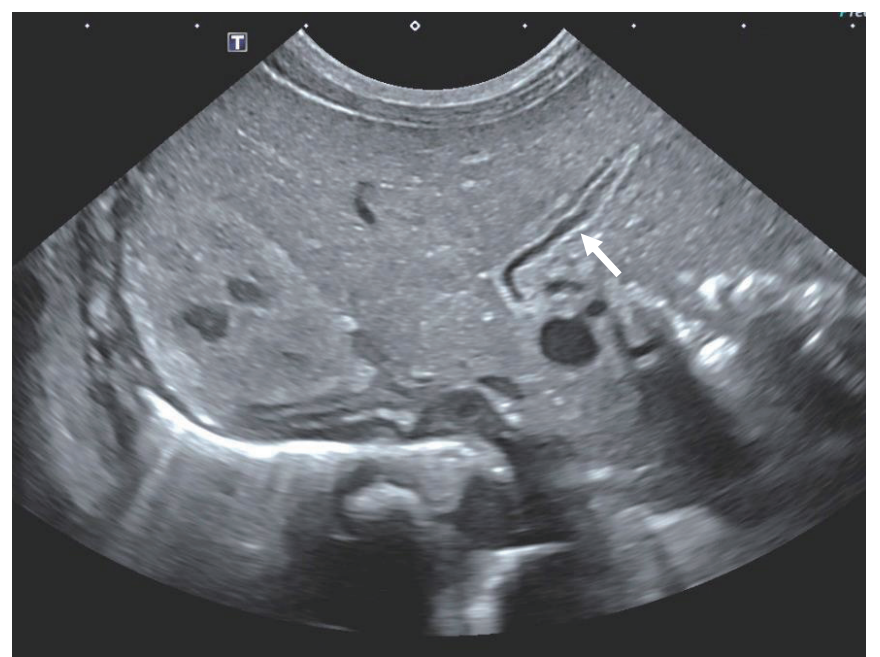

Figure 2. An abdominal ultrasonography suggestive of a biliary atresia. Abdominal ultrasonography at the 16th postnatal day showing a collapsed gallbladder strongly suggestive of congenital biliary atresia (arrow). 


\section{DISCUSSION}

Congenital pancreatic cysts are extremely rare in newborns; there are only 25 reported cases ${ }^{1,5}$. Pancreatic cysts are divided into six types: congenital-developmental, retention, duplication, pseudocysts, neoplastic, and parasitic ${ }^{2,7,8)}$. True pancreatic cysts are lined with true epithelium and include congenital develop mental cysts, retention cysts, and duplication cysts ${ }^{8)}$. Pseudocysts are the most common cysts of the pancreas. They develop after pancreatic fat necrosis is resorbed due to injury or inflammation of the pancreas. They are surrounded by granulation tissue and a fibrous capsule without an epithelial lining ${ }^{9}$. Neoplastic cysts can be either a mucinous type such as an intraductal papillary mucinous neoplasm, or a serous (clear-cell) type such as serous cystadenoma $^{9)}$.

Congenital pancreatic cysts are usually asymptomatic. However, they can present as an abdominal mass, abdominal distension, or vomiting due to a gastric outlet obstruction or cyst rupture. They can also present as cholangitis, pancreatitis, or peritonitis ${ }^{1,5,6,8)}$. Our patient did not present with any of the clinical symptoms previously reported.

The location of congenital pancreatic cysts are more commonly in the tail or neck of the pancreas (62\%), as opposed to the head $(32 \%)^{1,2,8)}$. However, $6 \%$ of the cysts are located in the overall pancreas $^{5)}$. In this reported case, the multifocal pancreatic cysts were located in both the head and tail of the pancreas.
Sepulveda et al. ${ }^{10)}$ reviewed eight reported cases of prenatally diagnosed congenital pancreatic cysts and found that the cyst is part of a systemic disease in five (63\%) out of eight cases. Congenital cysts may be associated with systemic diseases such as von Hippel-Lindau syndrome, polycystic kidney disease, Beckwith-Wiedemann syndrome, and asphyxiating thoracic dysplasia $^{5,6,9-11)}$. Therefore, clinicians should determine whether an associated systemic disease is present with the cyst. Clinicians also need to examine the kidneys, liver, bile duct, ovaries, mesentery, and urethra ${ }^{11)}$. In our case, the congenital pancreatic cyst was an isolated finding without other associated systemic diseases.

Radiologic findings and laboratory results are important when diagnosing a congenital pancreatic cyst ${ }^{7}$. Our case was diagnosed using MRCP. The most commonly used radiologic diagnostic tools include the abdominal computed tomography (CT) and abdominal magnetic resonance imaging $(\mathrm{MRI})^{7}$. However, some researchers have used an abdominal ultrasonography, and if there are any suspicious findings, a CT or MRI is also performed to confirm the diagnosis. Further, some researchers have aspirated the cystic fluid in adults, if the diagnosis was not certain based on radiologic findings ${ }^{12)}$. If the level of lipase in the cystic fluid is high, then the cyst can be considered to be a pancreatic cyst ${ }^{7}$.

Excision of the congenital pancreatic cyst is the treatment of choice. These type of cysts are usually located in the body or tail, allowing for complete excision to be easily performed ${ }^{3,11)}$. If the cyst is located in the tail, excision with distal pancreatectomy is

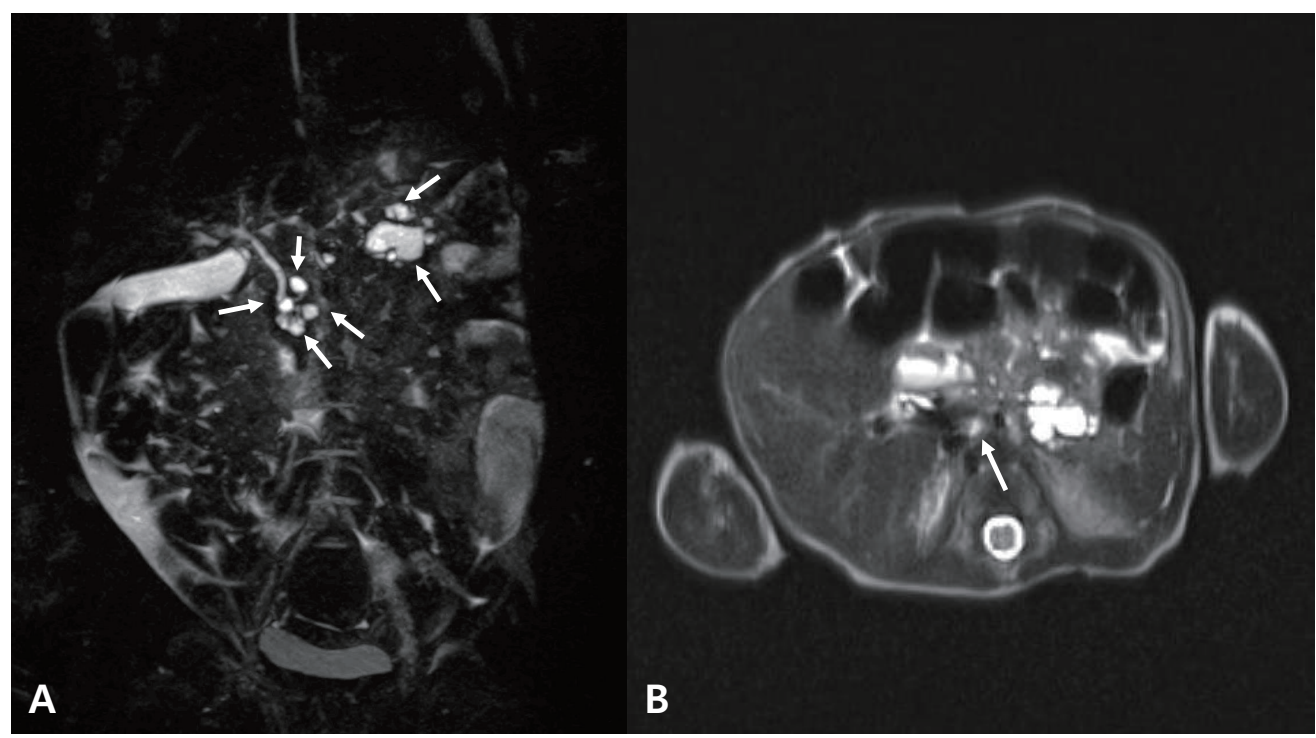

Figure 3. Magnetic resonance cholangio-pancreatography showing multiple pancreatic cysts. (A) There are multiple, variable sized cysts in the pancreatic head and tail (arrows). (B) The largest pancreatic cyst, $16 \mathrm{~mm}$ in diameter, located in the pancreatic tail (arrow). 
the preferred treatment. If the size of the cyst is increasing rapidly with clinical symptoms, prompt surgical intervention is needed ${ }^{3)}$. Unlike our case with multifocal cysts located in the pancreatic head and tail, Choi et al. ${ }^{13)}$ reported that an isolated $56 \times 47 \mathrm{~mm}$ homogenous cyst originating from the pancreatic tail was excised with a distal pancreatectomy. However, excision of the cyst is not always the treatment of choice for all patients. Treatment varies according to the location and size of the cyst ${ }^{5)}$. Warnock et al. ${ }^{5)}$ performed excision of multiple pancreatic cysts with distal pancreatectomy, because the baby had severe gastric outlet obstruction symptoms. Conversely, if multifocal cysts are present in the whole pancreas or if small cysts are located in the head, clinicians should consider waiting, with regular follow up with radiologic imaging, rather than performing immediate surgery. Our patient has multifocal cysts in the pancreatic head and tail, and a cystectomy or pancreatectomy has not been performed yet.

In conclusion, a congenital pancreatic cyst is an extremely rare lesion in newborns, and prenatal diagnosis is difficult due to this rarity. A congenital pancreatic cyst should be considered if the fetus has an abdominal cyst without a definite origin. A prompt diagnosis is crucial to prevent fatal complications.

\section{CONFLICT OF INTEREST}

No potential conflict of interest relevant to this article was reported.

\section{REFERENCES}

1. Auringer ST, Ulmer JL, Sumner TE, Turner CS. Congenital cyst of the pancreas. J Pediatr Surg 1993;28:1570-1.

2. Kazez A, Akpolat N, Kocakoc E, Parmaksiz ME, Koseogullari AA. Congenital true pancreatic cyst: a rare case. Diagn Interv Radiol 2006;12:31-3.

3. Castellani C, Zeder SL, Spuller E, Hollwarth ME. Neonatal congenital pancreatic cyst: diagnosis and management. J Pediatr Surg 2009;44:e1-4.

4. Boulanger SC, Gosche JR. Congenital pancreatic cyst arising from occlusion of the pancreatic duct. Pediatr Surg Int 2007;23: 903-5.

5. Warnock WT, Khoshnam N, Bird KM, Schaffer LR, Lou LH, Hawkins CM, et al. Congenital cyst of the pancreas: a case report and review of literature. Fetal Pediatr Pathol 2016;35:265-71.

6. Jamila C, Nahla K, Saida H, Sameh A, Amine K, Mongi M, et al. Neonatal congenital pancreatic cyst: a report of three cases. J Preg Child Health 2015;2:179.

7. Alzaiem M, Zaiem F, Zaiem F. Congenital pancreatic pseudocyst presenting as neonatal ascites. J Pediatr Surg Case Rep 2016;11: 44-6.

8. Bawazir OA, Al-Salem AH, Bawazir AO. Congenital pancreatic cyst: preoperative diagnosis and management. J Pediatr Surg Case Rep 2017;25:16-21.

9. Basturk O, Coban I, Adsay NV. Pancreatic cysts: pathologic classification, differential diagnosis, and clinical implications. Arch Pathol Lab Med 2009;133:423-38.

10. Sepulveda W, Carstens E, Sanchez J, Gutierrez J. Prenatal diagnosis of congenital pancreatic cyst: case report and review of the literature. J Ultrasound Med 2000;19:349-52.

11. Gerscovich EO, Jacoby B, Field NT, Sanchez T, Wootton-Gorges SL, Saroufeem R. Fetal true pancreatic cysts. J Ultrasound Med 2012;31:811-3.

12. Chak A. Endosonographic-guided therapy of pancreatic pseudocysts. Gastrointest Endosc 2000;52:S23-7.

13. Choi SJ, Kang MC, Kim YH, Lim JS, Lim SC, Chang JH. Prenatal detection of a congenital pancreatic cyst by ultrasound. J Korean Med Sci 2007;22:156-8. 